\title{
Neighbour isolated scattering number of graphs
}

\author{
Ersin Aslan \\ Turgutlu Vacational School, Celal Bayar University, Manisa, Turkey \\ e-mail: ersin.aslan@cbu.edu.tr
}

Received 31 Mar 2015

Accepted 15 Oct 2015

\begin{abstract}
The scattering number is a measure of the vulnerability of a graph. In this paper we investigate a refinement that involves the neighbour isolated version of the parameter. The neighbour isolated scattering number of a noncomplete graph $G$ is defined to be $\operatorname{NIS}(G)=\max \{i(G / X)-|X|: i(G / X) \geqslant 1\}$ where the maximum is taken over all $X$, the cut strategy of $G$, and $i(G / X)$ is the number of components which are isolated vertices of $G / X$. Like the scattering number itself, this is a measure of the vulnerability of a graph, but it is more sensitive. The relations between neighbour isolated scattering number and other parameters are determined and the neighbour isolated scattering number of trees and other families are obtained. We also give some results for the neighbour isolated scattering number of the graphs obtained by some graph operations.
\end{abstract}

KEYWORDS: graph theory, vulnerability, neighbour connectivity, neighbour scattering number

MSC2010: 05C40 68M10 68R10

\section{INTRODUCTION}

The problem of quantifying the vulnerability of graphs has received much attention recently, especially in the fields of computers, communication, and spy networks. In a network, the vulnerability parameters measure the resistance of the network to disruption of operation after the failure of certain stations or links. Parameters used to measure the vulnerability include connectivity, integrity ${ }^{1}$, scattering number ${ }^{2}$, isolated scattering number ${ }^{3}$, toughness ${ }^{4}$, tenacity ${ }^{5}$, and rupture degree ${ }^{6}$. However, most of these parameters do not consider the neighbourhoods of the effected vertices. On the other hand, in spy networks, if a spy or a station is captured, then adjacent stations are unreliable. Therefore neighbourhoods should be taken into consideration in spy networks. Nevertheless, there are very few parameters concerning neighbourhoods such as vertex neighbour connectivity ${ }^{7}$, vertex neighbour integrity ${ }^{8}$, and vertex neighbour scattering number ${ }^{9}$.

The scattering number of an incomplete connected graph $G$ is denoted and defined ${ }^{2}$ by

$$
\begin{array}{r}
s(G)=\max \{\omega(G-S)-|S|: S \subset V(G), \\
\omega(G-S) \geqslant 2\}
\end{array}
$$

where $\omega(G-S)$ denotes the number of components in $G-S$. Replacing $\omega(G-S)$ with $i(G-S)$ in the above definition gives ${ }^{3}$ the isolated scattering number, $I S(G)$, as a new parameter to measure the vulnerability of a network:

$$
I S(G)=\max \{i(G-S)-|S|: S \in C(G)\}
$$

where $G$ is a connected graph, $i(G-S)$ is the number of isolated vertices of $G-S$ and $C(G)$ is the set of vertex cuts of $G$.

The most common vulnerability parameters concerning with spy networks are as follows. The vertex neighbour connectivity of a graph $G$ is

$$
\operatorname{VNC}(G)=\min _{S \subseteq V(G)}\{|S|\}
$$

where $S$ is a subversion strategy of $G^{7}$. The vertex neighbour integrity of a graph $G$ is defined to be

$$
\operatorname{VNI}(G)=\min _{S \subseteq V(G)}\{|S|+m(G / S)\}
$$

where $S$ is any vertex subversion strategy of $G$ and $m(G / S)$ is the order of the largest connected component of $G / S^{8}$. The vertex neighbour scattering number of a graph $G$ is defined as

$$
\begin{aligned}
\operatorname{VNS}(G) & =\max \{\omega(G / X)-|X| \\
: X & \text { is a cut strategy of } G, \omega(G / X) \geqslant 1\},
\end{aligned}
$$

where $\omega(G / S)$ denotes the number of connected components in $G / X^{9}$.

Let $G$ be a finite simple graph with vertex set $V(G)$ and edge set $E(G)$. Let $\operatorname{deg}(v)$ denote the 
degree of the vertex $v$ in $G$. The set $N(v)=\{v \in$ $V(G) \mid v \neq u, v$ and $u$ are adjacent $\}$ is the open neighbourhood of $v$, and $N[v]=\{v\} \cup N(v)$ is the closed neighbourhood of $v$. A set of vertices $X \subseteq$ $V(G)$ is called a vertex subversion strategy of $G$ if each of the vertices in $X$ is subverted from $G$. By $G / X$ we denote the survival subgraph that remains after each vertex of $X$ is subverted from $G$. A vertex set $X$ is called a cut strategy of $G$ if the survival subgraph $G / X$ is disconnected, or is a clique, or is empty.

The known parameters concerning the neighbourhoods do not deal with the number of the removed vertices and the number of the components which are isolated vertices in a disrupted network simultaneously. In order to fill this void in the literature, the current study proposes a definition of neighbour isolated scattering number which is a new parameter concerning these two values. Motivated from the concept of the isolated scattering number and neighbour scattering number, it is natural for us to replace $\omega(G / X)$ with $i(G / X)$ in the above definition.

The neighbour isolated scattering number of a noncomplete graph $G$ is defined to be

$$
\operatorname{NIS}(G)=\max \{i(G / X)-|X|: i(G / X) \geqslant 1\}
$$

where the maximum is taken over all $X$, the cut strategy of $G$, and $i(G / X)$ is the number of components which are isolated vertices of $G / X$. A set $X \subset V(G)$ is said to be the NIS-set of $G$ if $\operatorname{NIS}(G)=$ $i(G / X)-|X|$. For the complete graph, subverting any one vertex will betray the entire graph, so we define $\operatorname{NIS}\left(K_{n}\right)=-1$.

The following examples show that the neighbour isolated scattering number is better than the vertex neighbour connectivity and the vertex neighbour integrity in measuring the vulnerability of graphs in some situations.

Example 1 It can be easily seen that the vertex neighbour connectivity of a path $P_{9}$ and a comet $C_{6,6}$ are equal: $\operatorname{VNC}\left(P_{9}\right)=\operatorname{VNC}\left(C_{6,6}\right)=1$.

Example 2 It can be easily seen that the vertex neighbour integrity of a cycle $C_{8}$ and a comet $C_{6,6}$ are equal: $\operatorname{VNI}\left(C_{8}\right)=\operatorname{VNI}\left(C_{6,6}\right)=3$. On the other hand, the neighbour isolated scattering numbers of a cycle $C_{8}$, a comet $C_{6,6}$, and a path $P_{9}$ are different: $\operatorname{NIS}\left(P_{9}\right)=1, \operatorname{NIS}\left(C_{8}\right)=0$, and $\operatorname{NIS}\left(C_{6,6}\right)=5$.

One finds that the neighbour isolated scattering number value of a graph is closely related to its vertex neighbour scattering number. But graphs with the same vertex neighbour scattering number may have different neighbour isolated scattering numbers.

Example 3 It can be easily seen that the vertex neighbour scattering number of a cycle $C_{9}$ and a wheel $W_{1,8}$ are equal: $\operatorname{VNS}\left(C_{9}\right)=\operatorname{VNS}\left(W_{1,8}\right)=0$. On the other hand, the neighbour isolated scattering number of a cycle $C_{9}$ and a wheel $W_{1,8}$ differ: $\operatorname{NIS}\left(C_{9}\right)=-1$ and $\operatorname{NIS}\left(W_{1,8}\right)=0$.

The definition of the neighbour isolated scattering number shows that the parameter measures not only the amount of work done to damage the network but also how badly the network is damaged. Graphs with a large neighbour isolated scattering number are more vulnerable.

\section{BOUNDS FOR NEIGHBOUR ISOLATED SCATTERING NUMBER}

In this section some lower and upper bounds are given for the neighbour isolated scattering number of a graph using different graph parameters.

Theorem 1 Let $G$ be a connected graph of order $n$. Then $\operatorname{NIS}(G) \geqslant 2-n$.

Proof: Let $X$ be a cut strategy of $G$. We have $|X| \leqslant$ $|N[X]|$ and $i(G / X) \geqslant 1$. Then

$$
n-|X| \geqslant n-|N[X]| \geqslant i(G / X) .
$$

If we add $i(G / X)$ to both sides, we have

$$
i(G / X)+n-|X| \geqslant 2 i(G / X) .
$$

Then, by the definition of neighbour isolated scattering number, $\operatorname{NIS}(G) \geqslant 2-n$.

Theorem 2 Let $G$ be a connected graph of order $n$. Then $\operatorname{NIS}(G) \leqslant n-2 \operatorname{VNC}(G)$.

Proof: Let $X$ be an NIS-set of $G$. For any set $X$ of $G$ we have $\operatorname{VNC}(G) \leqslant|X| \leqslant|N[X]|$ and $i(G / X) \leqslant$ $n-|N[X]|$. Thus

$$
i(G / X)-|X| \leqslant n-|N[X]|-|X| .
$$

Hence when we take the maximum of both sides, $\operatorname{NIS}(G) \leqslant n-2 \operatorname{VNC}(G)$.

Theorem 3 Let $G$ be a connected graph of order $n$ and $\delta(G)$ be the minimum vertex degree of $G$. Then

$$
\operatorname{NIS}(G) \leqslant n-\operatorname{VNC}(G)(\delta(G)+2) .
$$


Proof: Let $X$ be a NIS-set of $G$. For any set $X$ of $G$ we know $\operatorname{VNC}(G) \leqslant|X|$ and we have for any vertex, $v \in V(G),|N[v]| \geqslant \delta(G)+1$, and so $i(G / X) \leqslant n-$ $\operatorname{VNC}(G)(\delta(G)+1)$. Thus

$$
i(G / X)-|X| \leqslant n-\operatorname{VNC}(G)(\delta(G)+1)-\operatorname{VNC}(G) .
$$

Hence we get $\operatorname{NIS}(G) \leqslant n-\operatorname{VNC}(G)(\delta(G)+2)$.

Remark 1 The upper bound of NIS in Theorem 3 is tight. This can be shown by considering star graphs.

Theorem 4 Let $G$ be a connected graph and $\alpha(G)$ be the independence number of $G$. Then

$$
\operatorname{NIS}(G) \leqslant \alpha(G)-\operatorname{VNC}(G) .
$$

Proof: Let $X$ be a cut strategy of $G$. For any set $X$ of $G, \operatorname{VNC}(G) \leqslant|X|$ and $i(G / X) \leqslant \alpha(G)$. Hence we get $\operatorname{NIS}(G) \leqslant \alpha(G)-\operatorname{VNC}(G)$.

Theorem 5 Let $G$ be a connected graph. Then $\operatorname{NIS}(G) \geqslant 1-\operatorname{VNC}(G)$.

Proof: If $X$ is a cut strategy of $G$ with connectivity $\operatorname{VNC}(G)=|X|$ then $i(G / X) \geqslant 1$. Thus

$$
i(G / X)-|X| \geqslant 1-\operatorname{VNC}(G) .
$$

Hence we get $\operatorname{NIS}(G) \geqslant 1-\operatorname{VNC}(G)$.

Theorem 6 Let $G$ be a connected graph and $\delta(G)$ be the minimum vertex degree of $G$. Then $\operatorname{NIS}(G) \geqslant 1-$ $\delta(G)$.

Proof: By Theorem 5 we know $\operatorname{NIS}(G) \geqslant 1-$ $\operatorname{VNC}(G)$. We have $\delta(G) \geqslant \operatorname{VNC}(G)$ and so $\delta(G) \geqslant$ $\operatorname{VNC}(G) \geqslant 1-\operatorname{NIS}(G)$.

Remark 2 The lower bounds of NIS in Theorem 5 and Theorem 6 are tight. This can be shown by considering $P_{6}$ or $C_{7}$.

Theorem 7 Let $G$ be a connected graph. Then $\operatorname{NIS}(G) \leqslant \operatorname{VNS}(G)$.

Proof: If $X$ is a cut strategy of $G$, then $i(G / X) \leqslant$ $\omega(G / X)$. Hence $i(G / X)-|X| \leqslant \omega(G / X)-|X|$. Thus when we take the maximum of both sides, the proof is completed.

Remark 3 The upper bound of NIS in Theorem 7 is tight. This can be shown by considering $P_{12}$ or $W_{1,8}$.

For any graph $G$ the integrity ${ }^{1}$ of $G$ is denoted and defined by $I(G)=\min \{|S|+m(G-S): S \subseteq$ $V(G)\}$ where $m(G-S)$ denotes the maximum order (vertex-cardinality) of a component of graph $G-S$.
Lemma 1 (Ref. 10) For any graph $G, \operatorname{VNC}(G) \leqslant$ $\operatorname{VNI}(G)$.

Lemma 2 (Ref. 10) For any graph $G, \operatorname{VNI}(G) \leqslant$ $I(G)-r$ where $r$ is the maximum degree of the subgraph induced by an I-set of $G$.

The following results can be easily obtained from Theorem 5 and Lemmas 1 and 2.

Theorem 8 For any graph $G$, NIS $(G) \geqslant 1-\operatorname{VNI}(G)$.

Theorem 9 For any graph $G$, NIS $(G) \geqslant r+1-I(G)$, where $r$ is the maximum degree of the subgraph induced by an $I$-set of $G$.

\section{NEIGHBOUR ISOLATED SCATTERING NUMBER OF SEVERAL SPECIFIC CLASSES OF GRAPHS}

In this section, we consider the neighbour isolated scattering number of trees, path graphs, complete $k$-ary trees, comet graphs, cycle graphs, complete $k$ partite graphs, gear graphs, and star graphs.

Theorem 10 Let $T$ be a tree with order $n$. Then $0 \leqslant$ NIS $(T) \leqslant n-3$.

Proof: Let $X$ be a cut strategy of $T$. If $|X|=r$ then we have $i(T / X) \geqslant r$. Thus $i(T / X)-|X| \geqslant r-r$. Hence

$$
\operatorname{NIS}(T) \geqslant 0 \text {. }
$$

For any vertex, $v \in V(T),|N[v]| \geqslant 2$, so $i(T / X) \leqslant$ $n-2$. If $T$ is connected, then for any NIS-set $X, r \geqslant 1$ and $i(T / X) \leqslant n-2$. Hence $i(T / X)-|X| \leqslant n-2-r$. The function $f(r)=n-2-r$ is a decreasing function and it takes its maximum value at $r=1$. Hence we get

$$
\operatorname{NIS}(T) \leqslant n-3 .
$$

By (1) and (2), the proof is completed.

Theorem 11 Let $P_{n}$ be a path of order $n \geqslant 3$. Then

$$
\operatorname{NIS}\left(P_{n}\right)= \begin{cases}1, & n \equiv 1 \quad(\bmod 4) ; \\ 0, & n \equiv 0,2,3 \quad(\bmod 4) .\end{cases}
$$

Proof: Let $X$ be a cut strategy of $P_{n}$ and $|X|=r$. We distinguish two cases.

Case 1. If $n \equiv 1(\bmod 4)$, then $i\left(P_{n} / X\right) \leqslant r+1$. Thus $i\left(P_{n} / X\right)-|X| \leqslant r+1-r=1$. It can be easily seen that there is a cut strategy $X^{*}$ of $P_{n}$ such that $\left|X^{*}\right|=\frac{1}{4}(n-1), i\left(P_{n} / X^{*}\right)=\frac{1}{4}(n-1)+1$ and so

$$
\operatorname{NIS}\left(P_{n}\right)=1
$$

where $n \equiv 1(\bmod 4)$. 
Case 2. If $n \equiv 0,2,3(\bmod 4)$, then $i\left(P_{n} / X\right) \leqslant r$. Thus $i\left(P_{n} / X\right)-|X| \leqslant r-r=0$. It can be easily seen that there is a cut strategy $X^{*}$ of $P_{n}$ such that $\left|X^{*}\right|=$ $\left\lceil\frac{1}{4} n\right\rceil, i\left(P_{n} / X^{*}\right)=\left\lceil\frac{1}{4} n\right\rceil$ and so

$$
\operatorname{NIS}\left(P_{n}\right)=0
$$

where $n \equiv 0,2,3(\bmod 4)$. Hence by (3) and (4),

$$
\operatorname{NIS}\left(P_{n}\right)= \begin{cases}1, & n \equiv 1 \quad(\bmod 4) \\ 0, & n \equiv 0,2,3 \quad(\bmod 4)\end{cases}
$$

Theorem 12 Let $T_{k, d}$ be a complete $k$-ary tree of depth $d$ where $k \geqslant 2$. Then

$$
\begin{aligned}
& \operatorname{NIS}\left(T_{k, d}\right) \\
& =\left\{\begin{array}{lll}
\frac{k^{d+2}+1}{k^{2}+1}, & d \equiv 0 & (\bmod 4), \\
\frac{k^{d+2}-2 k^{2}+k-2}{k^{2}+1}, & d \equiv 1 & (\bmod 4), \\
\frac{k^{d+2}-1}{k^{2}+1}, & d \equiv 2 & (\bmod 4), \\
\frac{k^{d+4}-k^{d+2}-k^{3}+k}{k^{4}-1}, & d \equiv 3 & (\bmod 4) .
\end{array}\right.
\end{aligned}
$$

Proof: Let $X$ be a cut strategy of $T_{k, d}$ and let $|X|=$ $r$ be the number of removed vertices. The proof is similar to that of neighbour rupture degree of $T_{k, d}{ }^{11}$. There are four cases according to the depth of $T_{k, d}$.

Case $1: d \equiv 0(\bmod 4)$. (i) If $0 \leqslant r \leqslant k\left(k^{d}-\right.$ $1) /\left(k^{4}-1\right)$, then $i\left(T_{k, d} / X\right) \leqslant\left(k^{2}+k-1\right) r+1$. Thus $\operatorname{NIS}\left(T_{k, d}\right) \leqslant \max _{r}\left\{\left(k^{2}+k-1\right) r+1-r\right\}=\max _{r}\left\{\left(k^{2}+\right.\right.$ $k-2) r+1\}$. The function $f(r)=\left(k^{2}+k-2\right) r+1$ is an increasing function and it takes its maximum value at $r=k\left(k^{d}-1\right) /\left(k^{4}-1\right)$. Then

$$
\begin{aligned}
& \operatorname{NIS}\left(T_{k, d}\right) \\
& \quad \leqslant \frac{k^{d+3}+k^{d+2}-2 k^{d+1}+k^{4}-k^{3}-k^{2}+2 k-1}{k^{4}-1} .
\end{aligned}
$$

(ii) If $k\left(k^{d}-1\right) /\left(k^{4}-1\right)+1 \leqslant r \leqslant k^{2}\left(k^{d}-1\right) /\left(k^{4}-1\right)$, then $i\left(T_{k, d} / X\right) \leqslant k^{2}\left(k^{d}-1\right) /\left(k^{4}-1\right)+r\left(k^{2}-1\right)+1$. Then NIS $\left(T_{k, d}\right) \leqslant \max _{r}\left\{k^{2}\left(k^{d}-1\right) /\left(k^{4}-1\right)+r\left(k^{2}-\right.\right.$ $1)+1-r\}=\max _{r}\left\{k^{2}\left(k^{d}-1\right) /\left(k^{4}-1\right)+r\left(k^{2}-2\right)+1\right\}$. The function $f(r)=k^{2}\left(k^{d}-1\right) /\left(k^{4}-1\right)+r\left(k^{2}-2\right)+1$ is an increasing function and it takes its maximum value at $r=k^{2}\left(k^{d}-1\right) /\left(k^{4}-1\right)$. Hence

$$
\operatorname{NIS}\left(T_{k, d}\right) \leqslant \frac{k^{d+2}+1}{k^{2}+1} .
$$

(iii) If $k^{2}\left(k^{d}-1\right) /\left(k^{4}-1\right)+1 \leqslant r$, then we have $i\left(T_{k, d} / X\right) \leqslant\left(k^{d+4}-1\right) /\left(k^{4}-1\right)$. Thus

$$
\operatorname{NIS}\left(T_{k, d}\right) \leqslant \max _{r}\left\{\frac{k^{d+4}-1}{k^{4}-1}-r\right\} .
$$

The function $f(r)=\left(k^{d+4}-1\right) /\left(k^{4}-1\right)-r$ is a decreasing function and it takes its maximum value at $r=k^{2}\left(k^{d}-1\right) /\left(k^{4}-1\right)+1$. Then

$$
\operatorname{NIS}\left(T_{k, d}\right) \leqslant \frac{k^{d+4}-k^{d+2}-k^{4}+k^{2}}{k^{4}-1} .
$$

It can be easily seen that there is a cut strategy $X^{*}$ of $T_{k, d}$ such that $\left|X^{*}\right|=k^{2}\left(k^{d}-1\right) /\left(k^{4}-1\right)$ where $X^{*}$ contains all the vertices on the 2nd, 6th, 10th, 12th, etc, up to the $(d-2)$ th levels. Then $i\left(T_{k, d} / X^{*}\right)=$ $\left(k^{d+4}-1\right) /\left(k^{4}-1\right)$. Thus

$$
\operatorname{NIS}\left(T_{k, d}\right)=\frac{k^{d+2}+1}{k^{2}+1} .
$$

The proof is completed by (5), (6), (7) and (8).

Case $2: d \equiv 1(\bmod 4)$. (i) If $1 \leqslant r \leqslant k\left(k^{d}-\right.$ $k) /\left(k^{4}-1\right)$, then $i\left(T_{k, d} / X\right) \leqslant\left(k^{2}+k-1\right) r+1$. Thus $\operatorname{NIS}\left(T_{k, d}\right) \leqslant \max _{r}\left\{\left(k^{2}+k-1\right) r+1-r\right\}=\max _{r}\left\{\left(k^{2}+\right.\right.$ $k-2) r+1\}$. The function $f(r)=\left(k^{2}+k-2\right) r+1$ is an increasing function and it takes its maximum value at $r=k\left(k^{d}-k\right) /\left(k^{4}-1\right)$. Then

$\operatorname{NIS}\left(T_{k, d}\right)$

$$
\leqslant \frac{k^{d+3}+k^{d+2}-2 k^{d+1}-k^{3}+2 k^{2}-1}{k^{4}-1} .
$$

(ii) If $k\left(k^{d}-k\right) /\left(k^{4}-1\right)<r \leqslant k^{2}\left(k^{d}-k\right) /\left(k^{4}-1\right)$, then $i\left(T_{k, d} / X\right) \leqslant k^{2}\left(k^{d}-k\right) /\left(k^{4}-1\right)+\left(k^{2}-1\right)(r-1)$. Then

$$
\begin{aligned}
\operatorname{NIS}\left(T_{k, d}\right) & \leqslant \max _{r}\left\{\frac{k^{2}\left(k^{d}-k\right)}{k^{4}-1}+\left(k^{2}-1\right)(r-1)-r\right\} \\
& =\max _{r}\left\{\frac{k^{2}\left(k^{d}-k\right)}{k^{4}-1}+\left(k^{2}-2\right) r-k^{2}+1\right\} .
\end{aligned}
$$

The function $f(r)=k^{2}\left(k^{d}-k\right) /\left(k^{4}-1\right)+\left(k^{2}-\right.$ 2) $r-k^{2}+1$ is an increasing function and it takes its maximum value at $r=k^{2}\left(k^{d}-k\right) /\left(k^{4}-1\right)$. Hence

$$
\operatorname{NIS}\left(T_{k, d}\right) \leqslant \frac{k^{d+2}-k^{4}-k^{3}+1}{k^{2}+1} .
$$

(iii) If $r=k^{2}\left(k^{d}-k\right) /\left(k^{4}-1\right)+1$, then $i\left(T_{k, d} / X\right) \leqslant$ $\left(k^{d+4}-k^{4}-k+1\right) /\left(k^{4}-1\right)$. Hence

$$
\operatorname{NIS}\left(T_{k, d}\right) \leqslant \frac{k^{d+2}-2 k^{2}+k-2}{k^{2}+1} .
$$


(iv) If $k^{2}\left(k^{d}-k\right) /\left(k^{4}-1\right)+1<r$, then $i\left(T_{k, d} / X\right) \leqslant$ $\left(k^{d+4}-k^{4}-k+1\right) /\left(k^{4}-1\right)$. Then

$$
\operatorname{NIS}\left(T_{k, d}\right) \leqslant \max _{r}\left\{\frac{k^{d+4}-k^{4}-k+1}{k^{4}-1}-r\right\} .
$$

The function $f(r)=\left(k^{d+4}-k^{4}-k+1\right) /\left(k^{4}-1\right)-r$ is a decreasing function and it takes its maximum value at $r=k^{2}\left(k^{d}-1\right) /\left(k^{4}-1\right)+2$. Hence

$$
\operatorname{NIS}\left(T_{k, d}\right) \leqslant \frac{k^{d+2}-3 k^{2}+k-3}{k^{2}+1} .
$$

It is obvious that there is a cut strategy $X^{*}$ of $T_{k, d}$ such that $\left|X^{*}\right|=k^{2}\left(k^{d}-k\right) /\left(k^{4}-1\right)+1$ where $X^{*}$ contains all the vertices on the 3rd, 7th, 11th, etc., up to the $(d-2)$ th levels and one of the vertices on the first level. Then $i\left(T_{k, d} / X^{*}\right)=\left(k^{d+4}-k^{4}-k+\right.$ $1) /\left(k^{4}-1\right)$. Hence we get

$$
\operatorname{NIS}\left(T_{k, d}\right)=\frac{k^{d+2}-2 k^{2}+k-2}{k^{2}+1} .
$$

The proof is completed by (9), (10), (11), (12) and (13).

Case 3: $d \equiv 2(\bmod 4)$. (i) If $1 \leqslant r \leqslant k\left(k^{d}-\right.$ $\left.k^{2}\right) /\left(k^{4}-1\right)$, then $i\left(T_{k, d} / X\right) \leqslant\left(k^{2}+k-1\right) r+1$. Thus $\operatorname{NIS}\left(T_{k, d}\right) \leqslant \max _{r}\left\{\left(k^{2}+k-1\right) r+1-r\right\}=\max _{r}\left\{\left(k^{2}+\right.\right.$ $k-2) r+1\}$. The function $f(r)=\left(k^{2}+k-2\right) r+1$ is an increasing function and it takes its maximum value at $r=k\left(k^{d}-k^{2}\right) /\left(k^{4}-1\right)$. Then

$$
\begin{aligned}
& \operatorname{NIS}\left(T_{k, d}\right) \\
& \quad \leqslant \frac{k^{d+3}+k^{d+2}-2 k^{d+1}-k^{5}+2 k^{3}-1}{k^{4}-1} .
\end{aligned}
$$

(ii) If $r=k\left(k^{d}-k^{2}\right) /\left(k^{4}-1\right)+1$ then $i\left(T_{k, d} / X\right) \leqslant$ $\left(k^{2}+k-1\right) k\left(k^{d}-k^{2}\right) /\left(k^{4}-1\right)+k^{2}$. Then

$$
\operatorname{NIS}\left(T_{k, d}\right) \leqslant\left(k^{2}+k-1\right) \frac{k\left(k^{d}-k^{2}\right)}{k^{4}-1}+k^{2}-r
$$

and

$$
\begin{aligned}
& \operatorname{NIS}\left(T_{k, d}\right) \\
& \quad \leqslant \frac{k^{d}\left(k^{3}+k^{2}-2 k\right)+k^{6}-k^{5}-2 k^{4}+2 k^{3}-k^{2}+1}{k^{4}-1} .
\end{aligned}
$$

(iii) If $k\left(k^{d}-k^{2}\right) /\left(k^{4}-1\right)+1<r \leqslant\left(k^{d+2}-1\right) /\left(k^{4}-1\right)$, then $i\left(T_{k, d} / X\right) \leqslant\left(k^{d+2}-1\right) /\left(k^{4}-1\right)+\left(k^{2}-1\right) r$. Thus

$$
\operatorname{NIS}\left(T_{k, d}\right) \leqslant \max _{r}\left\{\frac{k^{d+2}-1}{k^{4}-1}+\left(k^{2}-1\right) r-r\right\} .
$$

The function $f(r)=\left(k^{d+2}-1\right) /\left(k^{4}-1\right)+\left(k^{2}-2\right) r$ is an increasing function and it takes its maximum value at $r=\left(k^{d+2}-1\right) /\left(k^{4}-1\right)$. Then

$$
\operatorname{NIS}\left(T_{k, d}\right) \leqslant \frac{k^{d+2}-1}{k^{2}+1} .
$$

(iv) If $\left(k^{d+2}-1\right) /\left(k^{4}-1\right)<r$, then $i\left(T_{k, d} / X\right) \leqslant$ $k^{2}\left(k^{d+2}-1\right) /\left(k^{4}-1\right)$. Then

$$
\operatorname{NIS}\left(T_{k, d}\right) \leqslant \max _{r}\left\{\frac{k^{2}\left(k^{d+2}-1\right)}{k^{4}-1}-r\right\} .
$$

The function $f(r)=k^{2}\left(k^{d+2}-1\right) /\left(k^{4}-1\right)-r$ is a decreasing function and it takes its maximum value at $r=\left(k^{d+2}-1\right) /\left(k^{4}-1\right)+1$. Hence

$$
\operatorname{NIS}\left(T_{k, d}\right) \leqslant \frac{k^{d+2}-k^{2}-2}{k^{2}+1} .
$$

It is obvious that there is a cut strategy $X^{*}$ of $T_{k, d}$ such that $\left|X^{*}\right|=\left(k^{d+2}-1\right) /\left(k^{4}-1\right)$ where $X^{*}$ contains all the vertices on the 0 th, 4 th, 8th, etc., up to the $(d-2)$ th levels. Then $i\left(T_{k, d} / X^{*}\right)=k^{2}\left(k^{d+2}-\right.$ $1) /\left(k^{4}-1\right)$. Hence we get

$$
\operatorname{NIS}\left(T_{k, d}\right)=\frac{k^{d+2}-1}{k^{2}+1} .
$$

The proof is completed by (14), (15), (16), (17), and (18).

Case 4: $d \equiv 3(\bmod 4)$. The proof is similar to those of Cases 1-3.

Theorem 13 Let $C_{m, n}$ be a comet with $m, n \geqslant 2$. Then

$$
\operatorname{NIS}\left(C_{m, n}\right)= \begin{cases}n, & m \equiv 0 \quad(\bmod 4), \\ n-1, & m \equiv 1,2,3 \quad(\bmod 4) .\end{cases}
$$

Proof: Suppose $V\left(P_{m}\right)=\left\{v_{1}, v_{2}, \ldots, v_{m}\right\}$ and $\operatorname{deg}\left(v_{1}\right)=n+1$. Let $X$ be a cut strategy of $C_{m, n}$ and $|X|=r$. We distinguish two cases.

Case 1 . If $m \equiv 0(\bmod 4)$ and $X=$ $\left\{v_{2}, v_{6}, \ldots, v_{m-2}\right\}$ then $i\left(C_{m, n} / X\right)=n+r$. So we have $i\left(C_{m, n} / X\right)-|X|=n+r-r$. Thus

$$
\operatorname{NIS}\left(C_{m, n}\right)=n \text {. }
$$

If $m \equiv 0(\bmod 4)$ and $X \neq\left\{v_{2}, v_{6}, \ldots, v_{m-2}\right\}$, then $i\left(C_{m, n} / X\right) \leqslant n+r$ and therefore

$$
i\left(C_{m, n} / X\right)-|X| \leqslant n+r-r
$$

and

$$
\operatorname{NIS}\left(C_{m, n}\right) \leqslant n
$$


Hence by (19) and (20), $\operatorname{NIS}\left(C_{m, n}\right)=n$ where $m \equiv 0$ $(\bmod 4)$.

Case 2 . If $m \equiv 1,2,3(\bmod 4)$ and $X=\left\{v_{2}\right\}$ then $C_{m, n} /\left\{v_{2}\right\}$ is a graph composed of a path of order $m-3$ and $n$ isolated vertices. So we have

$$
i\left(C_{m, n} / X\right)-|X|=n+r-1-r
$$

and

$$
\operatorname{NIS}\left(C_{m, n}\right)=n-1 .
$$

If $m \equiv 1,2,3(\bmod 4)$ and $X \neq\left\{v_{2}\right\}$, then $i\left(C_{m, n} / X\right) \leqslant n+r-1$. By the definition of neighbour isolated scattering number we have

$$
i\left(C_{m, n} / X\right)-|X| \leqslant n+r-1-r .
$$

Thus

$$
\operatorname{NIS}\left(C_{m, n}\right) \leqslant n-1 .
$$

Hence by (21) and (22), $\operatorname{NIS}\left(C_{m, n}\right)=n-1$ where $m \equiv 1,2,3(\bmod 4)$.

Theorem 14 Let $C_{n}$ be a cycle of order $n \geqslant 4$. Then

$$
\operatorname{NIS}\left(C_{n}\right)= \begin{cases}0, & n \equiv 0 \quad(\bmod 4), \\ -1, & n \equiv 1,2,3 \quad(\bmod 4) .\end{cases}
$$

Proof: Let $X$ be a cut strategy of $C_{n}$ and $|X|=r$ be the number of removed vertices of $C_{n}$. We have two cases to consider: $n \equiv 0(\bmod 4)$ and $n \equiv 1,2,3$ $(\bmod 4)$.

Case 1. If $n \equiv 0(\bmod 4)$, then $i\left(C_{n} / X\right) \leqslant r$. Hence $i\left(C_{n} / X\right)-|X| \leqslant r-r=0$. It can be easily seen that there is a cut strategy $X^{*}$ of $C_{n}$ such that $\left|X^{*}\right|=\frac{1}{4} n$ and $i\left(C_{n} / X^{*}\right)=\frac{1}{4} n$. Thus we get

$$
\operatorname{NIS}\left(C_{n}\right)=0
$$

where $n \equiv 0(\bmod 4)$.

Case 2. If $n \equiv 1,2,3(\bmod 4)$, then $i\left(C_{n} / X\right) \leqslant$ $r-1$. Thus $i\left(C_{n} / X\right)-|X| \leqslant r-1-r=-1$. It can be easily seen that there is a cut strategy $X^{*}$ of $C_{n}$ such that $|X|=\left\lceil\frac{1}{4} n\right\rceil$ and $i\left(C_{n} / X^{*}\right)=\left\lceil\frac{1}{4} n\right\rceil-1$. Therefore

$$
\operatorname{NIS}\left(C_{n}\right)=-1
$$

where $n \equiv 1,2,3(\bmod 4)$. The proof is completed from (23) and (24).

Theorem 15 Let $K_{n_{1}, n_{2}, \ldots, n_{k}}$ be a complete k-partite graph. Then

$$
\operatorname{NIS}\left(K_{n_{1}, n_{2}, \ldots, n_{k}}\right)=\max \left\{n_{1}, n_{2}, \ldots, n_{k}\right\}-2 .
$$

Proof: We assume $V\left(K_{n_{1}, n_{2}, \ldots, n_{k}}\right)=V_{1} \cup V_{2} \cup \ldots \cup V_{k}$ is a partition where $\left|V_{j}\right|=n_{j}$ for $j=1, \ldots, k$. Let $X$ be a cut strategy of $K_{n_{1}, n_{2}, \ldots, n_{k}}$ and let $X$ contain the elements which belongs to only one of the sets $V_{i}$. Otherwise $i\left(K_{n_{1}, n_{2}, \ldots, n_{k}} / X\right)=0$ and this contradicts the definition. If $X \subseteq V_{i}$ and $|X|=r$, then $i\left(K_{n_{1}, n_{2}, \ldots, n_{k}} / X\right)=n_{i}-r$. By the definition of the neighbour isolated scattering number, we get

$$
\operatorname{NIS}\left(K_{n_{1}, n_{2}, \ldots, n_{k}}\right)=\max _{r}\left\{n_{i}-2 r\right\} .
$$

The function $f(r)=n_{i}-2 r$ is a decreasing function and since $1 \leqslant r \leqslant n_{i}$ we have

$$
\operatorname{NIS}\left(K_{n_{1}, n_{2}, \ldots, n_{k}}\right)=n_{i}-2 .
$$

The proof is completed by taking $n_{i}=$ $\max \left\{n_{1}, n_{2}, \ldots, n_{k}\right\}$.

The following results can be easily obtained from Theorem 15.

Corollary 1 Let $K_{m, n}$ be a complete bipartite graph. Then

$$
\operatorname{NIS}\left(K_{m, n}\right)=\max \{m, n\}-2 .
$$

Corollary 2 Let $K_{1, n-1}$ be a star of order $n \geqslant 4$. Then

$$
\operatorname{NIS}\left(K_{1, n-1}\right)=n-3 .
$$

The gear graph ${ }^{12}$ is a wheel graph with a vertex added between each pair of adjacent graph vertices of the outer cycle. The gear graph $\mathrm{Ge}_{n}$ has $2 n+1$ vertices and $3 n$ edges.

Theorem 16 Let $\mathrm{Ge}_{n}$ be a gear graph. Then

$$
\operatorname{NIS}\left(\mathrm{Ge}_{n}\right)=n-1 \text {. }
$$

Proof: Let $X$ be a cut strategy of $\mathrm{Ge}_{n},|X|=r$, and $\operatorname{deg}(u)=n$. If $r \geqslant 1$ then we have $i\left(\mathrm{Ge}_{n} / X\right) \leqslant$ $n$. Hence $i\left(\mathrm{Ge}_{n} / X\right)-|X| \leqslant n-r$. The function $f(r)=n-r$ is a decreasing function and it takes its maximum value at $r=1$ and we have $\mathrm{NIS}\left(\mathrm{Ge}_{n}\right) \leqslant$ $n-1$. It is obvious that there is a cut strategy $X^{*}$ of $\mathrm{Ge}_{n}$ such that $X^{*}=\{u\}$. Then we have $i\left(\mathrm{Ge}_{n} / X\right)=$ $n$. Hence $\operatorname{NIS}\left(\mathrm{Ge}_{n}\right)=n-1$.

\section{GRAPH OPERATIONS AND NEIGHBOUR ISOLATED SCATTERING NUMBER}

In this section we consider results on the neighbour isolated scattering number of the join, union, corona, and Cartesian product of two graphs. 


\section{Join}

The join ${ }^{13} G=G_{1}+G_{2}$ has graph set $V(G)=V\left(G_{1}\right) \cup$ $V\left(G_{2}\right)$ and edge set $E(G)=E\left(G_{1}\right) \cup E\left(G_{2}\right) \cup\{u v \mid u \in$ $V\left(G_{1}\right)$ and $\left.v \in V\left(G_{2}\right)\right\}$.

Theorem 17 Let $G$ and $H$ be two connected graphs of order $m$ and $n$, respectively. Then

$$
\operatorname{NIS}(G+H)=\max \{\operatorname{NIS}(G), \operatorname{NIS}(H)\} .
$$

Proof: Let $X$ be a cut strategy of $G+H$ and $i((G+$ $H) / X) \geqslant 1$. Since every vertex of $G$ is adjacent to all vertices of $H$ and conversely, $X \subseteq V(G)$ and $X \cap$ $V(H)=\varnothing$ or $X \subseteq V(H)$ and $X \cap V(G)=\varnothing$. There are two cases according to the elements of $X$.

Case 1. Let $X=X_{1} \subseteq V(G)$ be the NIS-set of $G$ such that $\operatorname{NIS}(G)=i\left(G / X_{1}\right)-\left|X_{1}\right|$. Since every vertex of $G$ is adjacent to all vertices of $H$, we have

$$
\begin{aligned}
i\left((G+H) / X_{1}\right)-\left|X_{1}\right|=i\left(G / X_{1}\right) & -\left|X_{1}\right| \\
& =\operatorname{NIS}(G) .
\end{aligned}
$$

Case 2. Let $X=X_{2} \subseteq V(H)$ be the NIS-set of $H$ such that $\operatorname{NIS}(H)=i\left(H / X_{2}\right)-\left|X_{2}\right|$. Since every vertex of $H$ is adjacent to all vertices of $G$, we have

$$
i\left((G+H) / X_{2}\right)-\left|X_{2}\right|=i\left(H / X_{2}\right)-\left|X_{2}\right|=\operatorname{NIS}(H) .
$$

Hence by (25) and (26),

$$
\operatorname{NIS}(G+H)=\max \{\operatorname{NIS}(G), \operatorname{NIS}(H)\} .
$$

The following result can be easily obtained from Theorem 17.

Corollary 3 Let $W_{1, n}$ be a wheel, where $n \geqslant 4$. Then

$$
\operatorname{NIS}\left(W_{1, n}\right)= \begin{cases}0, & n \equiv 0 \quad(\bmod 4) \\ -1, & n \equiv 1,2,3 \quad(\bmod 4)\end{cases}
$$

\section{Union}

The union ${ }^{13}$ of $G_{1}$ and $G_{2}$ with disjoint vertex sets $V\left(G_{1}\right)$ and $V\left(G_{2}\right)$ and edge sets $E\left(G_{1}\right)$ and $E\left(G_{2}\right)$ is the graph $G=G_{1} \cup G_{2}$ with vertex set $V(G)=$ $V\left(G_{1}\right) \cup\left(G_{2}\right)$ and edge set $E(G)=E\left(G_{1}\right) \cup E\left(G_{2}\right)$.

Theorem 18 Let $G_{1}, G_{2}, \ldots, G_{n}$ be connected graphs. Then

$$
\begin{aligned}
\operatorname{NIS}\left(G_{1} \cup G_{2} \cup \ldots \cup G_{n}\right) & \\
& \geqslant \operatorname{NIS}\left(G_{1}\right)+\operatorname{NIS}\left(G_{2}\right)+\ldots+\operatorname{NIS}\left(G_{n}\right) .
\end{aligned}
$$

Proof: Let $X_{1}, X_{2}, \ldots, X_{n}$ be NIS-sets of $G_{1}, G_{2}, \ldots, G_{n}$, respectively, and let $X=$ $X_{1} \cup X_{2} \cup \ldots \cup X_{n}$ be a cut strategy of $G_{1} \cup G_{2} \cup \ldots \cup G_{n}$. By the definition of neighbour isolated scattering number we have

$$
\begin{aligned}
\operatorname{NIS} & \left(G_{1} \cup G_{2} \cup \ldots \cup G_{n}\right) \\
\geqslant & i\left(\left(G_{1} \cup G_{2} \cup \ldots \cup G_{n}\right) /\left(X_{1} \cup X_{2} \cup \ldots \cup X_{n}\right)\right) \\
& -\left|X_{1} \cup X_{2} \cup \ldots \cup X_{n}\right| \\
= & i\left(G_{1} / X_{1}\right)+i\left(G_{2} / X_{2}\right)+\ldots+i\left(G_{n} / X_{n}\right) \\
& -\left|X_{1}\right|-\left|X_{2}\right| \ldots-\left|X_{n}\right| \\
= & i\left(G_{1} / X_{1}\right)-\left|X_{1}\right|+i\left(G_{2} / X_{2}\right) \\
& -\left|X_{2}\right|+\ldots+i\left(G_{n} / X_{n}\right)-\left|X_{n}\right| \\
= & \operatorname{NIS}\left(G_{1}\right)+\operatorname{NIS}\left(G_{2}\right)+\ldots+\operatorname{NIS}\left(G_{n}\right) .
\end{aligned}
$$

Thus $\operatorname{NIS}\left(G_{1} \cup G_{2} \cup \ldots \cup G_{n}\right) \geqslant \operatorname{NIS}\left(G_{1}\right)+\operatorname{NIS}\left(G_{2}\right)+$ $\ldots+\operatorname{NIS}\left(G_{n}\right)$.

Theorem 19 Let $G$ and $H$ be two disjoint connected graphs. Then

$$
\begin{aligned}
\operatorname{NIS}(G \cup H)=\max \{\operatorname{NIS}(G)+ & \operatorname{NIS}(H), \\
& \operatorname{NIS}(G), \operatorname{NIS}(H)\} .
\end{aligned}
$$

Proof: The proof is similar to that of Theorem 18.

\section{Corona}

The corona ${ }^{13} G_{1} \circ G_{2}$ is defined as the graph $G$ obtained by taking one copy of $G_{1}$ of order $n$ and $n$ copies of $G_{2}$, and then joining the $i$ th vertex of $G_{1}$ to every vertex in the $i$ th copy of $G_{2}$.

Theorem 20 Let $G$ and $H$ be two connected graphs of order $m$ and $n$, respectively. Then

$$
\operatorname{NIS}(G \circ H)= \begin{cases}m \operatorname{NIS}(H), & \operatorname{NIS}(H) \geqslant 0, \\ \operatorname{NIS}(H), & \operatorname{NIS}(H)<0 .\end{cases}
$$

Proof: Let $X$ be a cut strategy of $G \circ H$ and $X_{1}$ be the NIS-set of $H$ such that NIS $(H)=i\left(H / X_{1}\right)-\left|X_{1}\right|$.

If $\operatorname{NIS}(H) \geqslant 0$, then $|X|=m\left|X_{1}\right|$ and $i((G \circ$ $H) / X)=m\left(i\left(H / X_{1}\right)\right)$. Thus $i((G \circ H) / X)-$ $|X|=m\left(i\left(H / X_{1}\right)\right)-m\left|X_{1}\right|=m\left(i\left(H / X_{1}\right)-\left|X_{1}\right|\right)=$ $m \mathrm{NIS}(H)$.

If $\operatorname{NIS}(H)<0$, then $|X|=\left|X_{1}\right|$ and $i((G \circ$ $H) / X)=i\left(H / X_{1}\right)$. Thus $i((G \circ H) / X)-|X|=$ $i\left(H / X_{1}\right)-\left|X_{1}\right|=\operatorname{NIS}(H)$.

The following results can be easily obtained from Theorem 20. 
Corollary 4 Let $G$ be a connected graph of order $m$ and $P_{n}$ be a path of order $n \geqslant 3$. Then

$$
\operatorname{NIS}\left(G \circ P_{n}\right)= \begin{cases}m, & n \equiv 1 \quad(\bmod 4), \\ 0, & n \equiv 0,2,3(\bmod 4) .\end{cases}
$$

Corollary 5 Let $G$ be a connected graph of order $m$ and $C_{n}$ be a cycle of order $n \geqslant 4$. Then

$$
\operatorname{NIS}\left(G \circ C_{n}\right)= \begin{cases}0, & n \equiv 0 \quad(\bmod 4), \\ -1, & n \equiv 1,2,3 \quad(\bmod 4) .\end{cases}
$$

\section{Cartesian product}

The Cartesian product ${ }^{13} G_{1} \times G_{2}$ of graphs $G_{1}$ and $G_{2}$ has $V\left(G_{1}\right) \times V\left(G_{2}\right)$ as its vertex set and $\left(u_{1}, u_{2}\right)$ is adjacent to $\left(v_{1}, v_{2}\right)$ if either $u_{1}=v_{1}$ and $u_{2}$ is adjacent to $v_{2}$ or $u_{2}=v_{2}$ and $u_{1}$ is adjacent to $v_{1}$.

Theorem 21 Let $G$ and $H$ be two disjoint connected graphs. Then

$$
\operatorname{NIS}(G \times H) \geqslant \max \{\operatorname{NIS}(G), \operatorname{NIS}(H)\}
$$

Proof: The proof is similar to that of Theorem 17 and 18.

Corollary 6 Let $G$ be a connected graph of order $m$ and $P_{n}$ be a path of order $n \geqslant 3$. Then

$$
\operatorname{NIS}\left(K_{2} \times P_{n}\right)=\left\{\begin{array}{lll}
\frac{1}{3} n, & n \equiv 0 & (\bmod 3), \\
\frac{1}{3}(n-4), & n \equiv 1 \quad(\bmod 3), \\
\frac{1}{3}(n-2), & n \equiv 2(\bmod 3) .
\end{array}\right.
$$

Proof: Let $X$ be a cut strategy of $K_{2} \times P_{n}$ and $|X|=r$. We distinguish three cases.

Case $1: n \equiv 0(\bmod 3)$. If $1 \leqslant r \leqslant \frac{1}{3} n$, then $i\left(\left(K_{2} \times P_{n}\right) / X\right) \leqslant 2 r$. Thus

$$
i\left(\left(K_{2} \times P_{n}\right) / X\right)-|X| \leqslant 2 r-r=r .
$$

The function $f(r)=r$ is an increasing function and it takes its maximum value at $r=\frac{1}{3} n$.

$$
\operatorname{NIS}\left(K_{2} \times P_{n}\right) \leqslant \frac{n}{3} .
$$

$$
\text { If } \frac{1}{3} n<r \leqslant 2 n \text {, then } i\left(\left(K_{2} \times P_{n}\right) / X\right) \leqslant n-r \text {. Thus }
$$

$$
i\left(\left(K_{2} \times P_{n}\right) / X\right)-|X| \leqslant n-r-r=n-2 r .
$$

The function $f(r)=n-2 r$ is a decreasing function and it takes its maximum value at $r=\frac{1}{3} n+1$.

$$
\operatorname{NIS}\left(K_{2} \times P_{n}\right) \leqslant \frac{n}{3}-2 .
$$

It can be easily seen that there is a cut strategy $X^{*}$ of $\left(K_{2} \times P_{n}\right)$ such that $\left|X^{*}\right|=\frac{1}{3} n, i\left(\left(K_{2} \times P_{n}\right) / X^{*}\right)=\frac{1}{3} 2 n$ and so

$$
\operatorname{NIS}\left(K_{2} \times P_{n}\right)=\frac{n}{3} .
$$

By (27), (28), and (29) we have

$$
\operatorname{NIS}\left(K_{2} \times P_{n}\right)=\frac{n}{3},
$$

where $n \equiv 0(\bmod 3)$.

Case 2: $n \equiv 1(\bmod 3)$. If $1 \leqslant r \leqslant \frac{1}{3}(n-1)+1$ then $i\left(\left(K_{2} \times P_{n}\right) / X\right) \leqslant 2 r-2$. Thus

$$
i\left(\left(K_{2} \times P_{n}\right) / X\right)-|X| \leqslant 2 r-2-r=r-2 .
$$

The function $f(r)=r-2$ is an increasing function and it takes its maximum value at $r=\frac{1}{3}(n-1)+1$.

$$
\operatorname{NIS}\left(K_{2} \times P_{n}\right) \leqslant \frac{n-4}{3} .
$$

If $\frac{1}{3}(n-1)+1<r \leqslant 2 n$, then $i\left(\left(K_{2} \times P_{n}\right) / X\right) \leqslant n-r$. Thus

$$
i\left(\left(K_{2} \times P_{n}\right) / X\right)-|X| \leqslant n-r+1-r=n-2 r+1 .
$$

The function $f(r)=n-2 r+1$ is a decreasing function and it takes its maximum value at $r=\frac{1}{3}(n+5)$.

$$
\operatorname{NIS}\left(K_{2} \times P_{n}\right) \leqslant \frac{n-7}{3} .
$$

It is obvious that there exists $X^{*}$ of $\left(K_{2} \times P_{n}\right)$ such that $\left|X^{*}\right|=\frac{1}{3}(n-1)+1, i\left(\left(K_{2} \times P_{n}\right) / X^{*}\right)=\frac{1}{3}(2 n-2)$ and so

$$
\operatorname{NIS}\left(K_{2} \times P_{n}\right)=\frac{n-4}{3} .
$$

By (31), (32), and (33) we have

$$
\operatorname{NIS}\left(K_{2} \times P_{n}\right)=\frac{n-4}{3},
$$

where $n \equiv 1(\bmod 3)$.

Case $3: n \equiv 2(\bmod 3)$. If $1 \leqslant r \leqslant \frac{1}{3}(n+1)$ then $i\left(\left(K_{2} \times P_{n}\right) / X\right) \leqslant 2 r-1$. Thus

$$
i\left(\left(K_{2} \times P_{n}\right) / X\right)-|X| \leqslant 2 r-1-r=r-1 .
$$

The function $f(r)=r$ is an increasing function and it takes its maximum value at $r=\frac{1}{3}(n+1)$.

$$
\operatorname{NIS}\left(K_{2} \times P_{n}\right) \leqslant \frac{n-2}{3} .
$$

If $\frac{1}{3}(n+1)<r \leqslant 2 n$ then $i\left(\left(K_{2} \times P_{n}\right) / X\right) \leqslant n-r+1$. Thus

$$
i\left(\left(K_{2} \times P_{n}\right) / X\right)-|X| \leqslant n-r+1-r=n-2 r+1 .
$$


The function $f(r)=n-2 r+1$ is a decreasing function and it takes its maximum value at $r=\frac{1}{3}(n+4)$.

$$
\operatorname{NIS}\left(K_{2} \times P_{n}\right) \leqslant \frac{n-5}{3} .
$$

It can be easily seen that there is a cut strategy $X^{*}$ of $\left(K_{2} \times P_{n}\right)$ such that $\left|X^{*}\right|=\frac{1}{3} n, i\left(\left(K_{2} \times P_{n}\right) / X^{*}\right)=$ $\frac{1}{3}(2 n-1)$ and so

$$
\operatorname{NIS}\left(K_{2} \times P_{n}\right)=\frac{n-2}{3} .
$$

By (35), (36), and (37) we have

$$
\operatorname{NIS}\left(K_{2} \times P_{n}\right)=\frac{n-2}{3},
$$

where $n \equiv 0(\bmod 3)$. By (30), (34), and (38) we have

$$
\operatorname{NIS}\left(K_{2} \times P_{n}\right)=\left\{\begin{array}{lll}
\frac{1}{3} n, & n \equiv 0 & (\bmod 3) \\
\frac{1}{3}(n-4), & n \equiv 1 & (\bmod 3) \\
\frac{1}{3}(n-2), & n \equiv 2 & (\bmod 3)
\end{array}\right.
$$

Corollary 7 Let $P_{n}$ be a path of order $n \geqslant 3$. Then

$$
\operatorname{NIS}\left(P_{3} \times P_{n}\right)=\left\{\begin{array}{lll}
\frac{1}{2} n, & n \equiv 0 & (\bmod 2), \\
3+\frac{1}{2}(n-3), & n \equiv 1 & (\bmod 2) .
\end{array}\right.
$$

\section{CONCLUSIONS}

We investigated a new measure for reliability of a graph called the neighbour isolated scattering number. The vertex neighbour connectivity is sensitive to the number of edges present in a graph, and the vertex neighbour connectivity or vertex neighbour integrity or vertex neighbour scattering number cannot distinguish the vulnerability of different networks very well in some situations. If we want to choose the more stable graph among the graphs which have the same order and the same size, one way is to choose the graph whose neighbour isolated scattering number is less than those of the others.

Acknowledgements: The author is thankful to anonymous referees for their constructive suggestions and critical comments, which led to this improved version.

\section{REFERENCES}

1. Barefoot CA, Etringer R, Swart HC (1987) Vulnerability in graphs - a comparative survey. $J$ Combin Math Combin Comput 1, 13-22.

2. Jung H (1978) On a class of posets and the corresponding comparability graphs. $J$ Combin Theor $B$ 1124, 125-33.
3. Wang SY, Yang YX, Lin SW, Li J, Hu ZM (2011) The isolated scattering number of graphs. Acta Math Sin 54, 861-74.

4. Chvátal V (1973) Tough graphs and hamiltonian circuits. Discrete Math 306, 215-28.

5. Cozzens MB, Moazzami D, Stueckle S (1994) The tenacity of the Harary graphs. $J$ Combin Math Combin Comput 16, 33-56.

6. Li Y, Zhang S, Li X (2005) Rupture degree of graphs. Int $J$ Comput Math 82, 793-803.

7. Gunther G (1985) Neighbor connectivity in regular graphs. Discrete Appl Math 11, 233-43.

8. Cozzens MB, Wu SSY (1996) Vertex-neighborintegrity of trees. Ars Combinatoria 43, 169-80.

9. Wei ZT, Mai A, Zhai M (2011) Vertex-neighborscattering number of graphs. Ars Combinatoria 102, 417-26.

10. Wu SSY, Cozzens MB (2000) Relationships between vertex-neighbor-integrity and other parameters. Ars Combinatoria 55, 271-82.

11. Bacak-Turan G, Kirlangic A (2011) Neighbor rupture degree and the relations between other parameters. Ars Combinatoria 102, 333-52.

12. Brandstädt A, Le VB, Spinrad JP (1999) Graph Classes: A Survey, SIAM Monographs on Discrete Mathematics and Applications, Springer.

13. Chartrand G, Lesniak I (1996) Graphs and Digraphs, 3rd edn, Chapman Hall, Orange, California. 